\title{
Comparison of three types of central venous catheters in patients with malignant tumor receiving chemotherapy
}

This article was published in the following Dove Press journal:

Patient Preference and Adherence

12 July 2017

Number of times this article has been viewed

\author{
Shirong Fang' \\ Jinhong Yang ${ }^{2}$ \\ Lei Song ${ }^{3}$ \\ Yan Jiang' \\ Yuxiu $\mathrm{Liu}^{4}$ \\ 'Department of Anesthesiology, \\ ${ }^{2}$ Department of Oncology, Weifang \\ People's Hospital, Weifang, 'Intensive \\ Care Unit, The Affiliated Hospital of \\ Qingdao University, Qingdao, ${ }^{4}$ Nursing \\ College, Weifang Medical University, \\ Weifang, People's Republic of China
}

Correspondence: Yuxiu Liu Nursing College, Weifang Medical University, No 7166 Baotong West Road, Weicheng District, Weifang 26600I, People's Republic of China

Tel +86 18663608162

Email18663608I62@I63.com
Background: Central venous catheters (CVCs) have been an effective access for chemotherapy instead of peripherally intravenous catheters. There were limited studies on the choices and effects of different types of CVCs for chemotherapy. The aim of this study was to compare the complications, cost, and patients' quality of life and satisfaction of three commonly used CVCs for chemotherapy, such as implanted venous port, peripherally inserted central catheters (PICCs), and external non-tunneled central venous catheters (NTCs).

Methods: A double-center prospective cohort study was carried out from March 2014 to December 2016. Catheterization situation, complications, catheter maintenance, cost, and patients' quality of life and satisfaction were recorded, investigated, and analyzed. Forty-five ports, 60 PICCs and 40 NTCs were included. All the CVCs were followed up to catheter removal.

Results: There was no statistical difference in catheterization success rates between port and PICC. NTC had less success rate by one puncture compared with port. Ports had fewer complications compared with PICCs and NTCs. The complication rates of ports, PICCs and NTCs were $2.2 \%, 40 \%$, and $27.5 \%$, respectively. If the chemotherapy process was $<12$ months, NTCs cost least, and the cost of port was much higher than PICC and NTC. When the duration time was longer than 12 months, the cost of port had no difference with the cost of PICC. Quality of life and patients' satisfaction of port group were significantly higher than the other two groups

Conclusion: Although port catheterization costs more and needs professional medical staff and strict operational conditions, ports have fewer complications and higher quality of life and patients' satisfaction than PICCs and NTCs. Therefore, not following consideration of the economic factor, we recommend port as a safe and an effective chemotherapy access for cancer patients, especially for whom needing long chemotherapy process.

Keywords: central venous catheter, port, peripherally inserted central catheter, external nontunneled catheter, complication, cost, cancer patient

\section{Introduction}

Central venous catheters (CVCs) have been largely used in the oncology department in People's Republic of China. The use of CVCs protects the peripheral veins and offers an effective access for chemotherapy. Especially for chemotherapy regimens containing prolonged infusion of 5-fluorouracil (5-FU), CVCs represent obvious advantage than peripheral vein catheters. ${ }^{1}$

CVCs include implantable central venous ports (ports), peripherally inserted central catheters (PICCs), and external tunneled or NTCs (external CVCs). ${ }^{2}$ PICCs are most widely used in the field of cancer chemotherapy in China. In recent years, 
the use of ports has increased considerably. Other tunneled or non-tunneled CVCs (NTCs) in the internal jugular vein or subclavian vein have also been used as central venous access for chemotherapy, of which the NTCs are more frequently used. Implantable ports have been reported to have significantly lower infection rates compared with other central venous access devices. ${ }^{3}$ Patients with ports can conduct activities such as swimming and have more positivity in terms of body image than other CVCs. ${ }^{4}$ PICCs offer many advantages such as avoiding CVC placement-associated mechanical complications such as pneumothorax and hemorrhage, and the professional nurse PICC teams have made their use more accessible in oncology departments. ${ }^{5}$ In China, the NTCs in the internal jugular vein or subclavian vein cost less than other CVCs and are used when the chemotherapy process is short. Complications of NTCs were reported more than PICCs and ports. ${ }^{6-8}$ But in Leon's study, no significant differences were found when complication rates in PICC and non-tunneled devices were compared. ${ }^{9}$ According to the Centers for Disease Control and Prevention (CDC) guideline, PICCs had lower rate of infection than NTCs, and totally implantable catheter had the lowest risk for catheterrelated bloodstream infection (CRBSI). ${ }^{10}$ The systematic review of Chopra et $\mathrm{al}^{11}$ found that although PICCs were associated with a lower risk of CRBSI than other CVCs in outpatients, hospitalized patients might be just as likely to experience CRBSI with PICCs as with other CVCs. There is no consistent evidence to guide which type of CVC is safer or preferable for cancer patients. ${ }^{1}$ Furthermore, few studies have actually assessed the differences between these three types of CVCs.

In order to give evidence for clinical patient selection of CVCs for chemotherapy, we conducted a prospective double-center cohort study to compare the complications, cost, and patients' preference among ports, PICCs and NTCs in patients with malignant tumor.

\section{Methods}

\section{Patients and data collection}

From March 2014 to December 2016, a prospective cohort study was carried out in Weifang People's Hospital and The Affiliated Hospital of Qingdao University. Both hospitals are public tertiary comprehensive hospitals. Inclusion criteria were as follows: 1 ) patients aged $>18$ years with malignant tumor confirmed by pathology; 2) patients planned for chemotherapy by a CVC; and 3) patients without chemotherapy and venous catheterization contraindications. Ethical approval was obtained from the Ethics Committee of Weifang People's
Hospital and the Ethics Committee of the Affiliated Hospital of Qingdao University, and all patients signed written informed consent before inclusion in the study.

The data collected contained the following: 1) patients' general information; 2) diagnosis and treatment data; 3) CVC catheterization records; 4) complication records; 5) costs of different types of CVCs; 5) catheter removal time and reason; and 6) patients' quality of life, comfort, and satisfaction with CVC. Patients' general information, diagnosis, and treatment data were collected from medical records. CVC catheterization information was recorded by the authors or searched by electronic medical records. Complications of different types of CVCs were collected by weekly maintenance or follow-up. The costs contained the costs of CVC catheterization, maintenance, complication treatment, and removal cost. Maintenance- and complication-associated costs were calculated according to CVC indwelling time and recorded complications. Costs were calculated in Chinese RMB and converted to US dollars using a standard rate of 6.87:1. Quality of life and comfort with CVC were investigated by a questionnaire modified by three-round expert inquiry. The questionnaire included eight aspects: sleep quality, psychological status, personal image, clothing, complications worrying, activities of daily life, social life, and work activities. Every aspect got a score from 1 to 4 , 1 stood for the worst condition and 4 stood for the best condition of this aspect. The score of quality of life and comfort ranged from 8 to 32 . If the score was $<16$, the patient was recorded discomfort. If the score was 16-24, medium comfort was recorded. If the score was $>24$, comfort was recorded. Patients were asked about the questionnaire after 2 weeks of insertion and at the time of catheter removal, and the average score was analyzed. The satisfactory information was collected by an open-ended questionnaire, divided into two grades, not satisfied and satisfied. The satisfaction questionnaire was asked at the time of catheter removal. All the CVCs were followed up to catheter removal. Follow-up started from the day of CVC catheterization until catheter removal.

\section{Catheterization and maintenance of different types of CVCs}

Port implantation was done by well-trained surgeons in the operating room. 7 Fr Groshong ${ }^{\circledR}$ single-lumen Bard Port was used in the study. Patients took the supine position with head turning to opposite side of the operation and shoulder padded high. Under local anesthesia $(10 \mathrm{~mL}$ of $2 \%$ mepivacaine hydrochloride), the catheter was placed into the jugular vein or subclavian vein with puncture point $1-2 \mathrm{~cm}$ below the 
middle point between the cleidomastoid sternal branch and top or clavicle at the clavicle angle. The port was embedded in the subclavian fossa in 1-2 cm of subcutaneous tissue, and then the catheter and port were fixedly connected by the catheter lock. A routine chest X-ray film was examined for the catheter position. ${ }^{12,13}$ For maintenance, Huber needle was used to puncture into the port after skin disinfection and normal saline were used to flush the catheter. When in use, Huber needle should be replaced every week, and when not in use, the port should be washed every 4 weeks. After completion of the infusion therapy, $10 \mathrm{~mL}$ heparin saline $(100 \mathrm{IU} / \mathrm{mL})$ was used to seal the catheter.

PICC was performed by professional PICC nurses in a clean separated ward. 4 Fr single-lumen Bard Groshong PICC was used in the study. PICC could be inserted by cephalic veins, basilic veins, and venae median cubiti, of which basilic veins were the first choice. Chest radiography was routinely examined to confirm the catheter tip position. The PICC was flushed with $10 \mathrm{~mL}$ saline after catheterization, after each use and once a week between chemotherapy. The catheter entrance site was covered with dressing and changed every week after skin disinfection. ${ }^{5}$

NTC was inserted by physicians in a clean ward. A $16 \mathrm{G}$ single-lumen Arrow CVC was used in the study. After local anesthesia, the catheter was placed into the jugular vein or subclavian vein with puncture needle point along with sternoclavicular joint as the needle direction by the Seldinger technique. The maintenance method of NTC was similar to PICC.

\section{Complications}

The complications in the study contained catheterization complication, CRBSI, local infection, catheter-related thrombosis, mechanical phlebitis, malposition, catheter breakage, catheter occlusion, and pinch-off syndrome. Catheterization complication was that happening in a consequence of the catheter insertion procedure such as pneumothorax, arrhythmias, and major bleeding. CRBSI was defined as when the same organism was isolated from the peripheral blood as from the catheter in a patient with clinical signs of infection (fever $>38^{\circ} \mathrm{C}$, chills, rigor, hypotension) and no other sources of infection. ${ }^{10,14}$ Local infection was defined as catheter insertion local exit-site infection. Catheter-related thrombosis was diagnosed by ultrasound with clinical symptoms or signs of venous thrombosis. ${ }^{15}$ Mechanical phlebitis was defined as at least one sign or symptom of vein irritation confined to the CVC insertion site. ${ }^{16}$ Malposition was described as displacement or migration of the $\mathrm{CVC}$ from its original site.
Catheter occlusion was identified as impossible to infuse fluids into the catheter or to withdraw blood from it without any medical treatment. Pinch-off syndrome was defined that the catheter became kinked, compressed, or even fragmented at the narrow space between the clavicle and the first rib. ${ }^{17}$

\section{Statistical analysis}

The data were recorded and analyzed using SPSS 19.0. Continuous variables were presented as mean \pm standard deviation. Chi-square test was used to evaluate the difference of enumeration data. Analysis of variance (ANOVA) was used to compare the difference of three groups for measurement data. Dunnett's $t$-test was used for the comparison of two averages. Kaplan-Meier survival analysis was used to visualize the catheter duration rate over time. The significance level was set at 0.05 .

\section{Results \\ Patient characteristics}

During our study period, 45 ports, 60 PICCs, and 40 NTCs were followed up to catheter removal. No patient had multiple CVCs. Of the patients, 59 were male and 86 were female, and the average age was 52.42 years (range 20-72 years). Of the included patients, 56 patients were diagnosed with breast cancer, 42 were lung cancer, 38 were gastrointestinal (GI) malignancy, and 9 were other cancer cases, including 5 ovarian cancer cases, 2 nasopharyngeal carcinoma cases, and 2 liver cancer cases. The baseline patient characteristics were not statistically different among the three groups (Table 1).

\section{Data related to PICC placement}

Forty-three port catheterizations were successful by only one puncture, with one puncture success rate of $95.6 \%$. Fifty-four PICCs were inserted by only one puncture, with one puncture success rate of $90 \%(P>0.05$ compared with port). Thirty-two NTCs were placed by one puncture $(80 \%)$ ( $P>0.05$ compared with PICC, $P<0.05$ compared with port). The mean duration time of ports was $338.3 \pm 93.8$ days (152-527 days), PICC 261.1 \pm 63.6 days (148-450 days) and 119.9 \pm 33.2 days for NTCs $(65-196$ days $)(P<0.01)$. The mean duration time of ports was significantly longer than PICCs and NTCs $(P<0.01$ and $P<0.01$, respectively). PICCs had longer indwelling time than NTCs $(P<0.01)$. The detailed time duration of three types of CVCs is shown in Table 2.

Forty-two ports were removed due to completion of therapy, two were because of patient death, and one was removed due to catheter occlusion. Fifty-six PICCs were 
Table I Patient demographic characteristics of three types of CVCs

\begin{tabular}{|c|c|c|c|c|}
\hline Variables & Port & PICC & NTC & $P$-value \\
\hline Median age, years & 52.20 & 52.38 & 51.73 & 0.978 \\
\hline Gender & & & & 0.663 \\
\hline Male & 20 & 25 & 14 & \\
\hline Female & 25 & 35 & 26 & \\
\hline Cancer diagnosis & & & & 0.801 \\
\hline Breast cancer & 18 & 22 & 16 & \\
\hline Lung cancer & 12 & 18 & 12 & \\
\hline Gl cancer & 13 & 14 & 11 & \\
\hline Others & 2 & 6 & 1 & \\
\hline Cancer stage & & & & 0.355 \\
\hline I & 0 & 2 & 2 & \\
\hline II & 7 & 14 & 12 & \\
\hline III & 23 & 21 & 13 & \\
\hline IV & 25 & 23 & 13 & \\
\hline \multicolumn{3}{|c|}{ Chemotherapeutic agent } & & 0.541 \\
\hline CAF & 2 & 4 & 1 & \\
\hline DCF & 2 & 6 & 1 & \\
\hline FOLFIRI & 4 & 4 & 2 & \\
\hline FOLFOX & 7 & 6 & 4 & \\
\hline GP & 3 & 4 & 4 & \\
\hline GT & 3 & 1 & 0 & \\
\hline NP & 3 & 8 & 1 & \\
\hline $\mathrm{PF}$ & 0 & 1 & 1 & \\
\hline PP & 2 & 6 & 3 & \\
\hline TAC & 10 & 10 & 9 & \\
\hline $\mathrm{TF}$ & 0 & 0 & I & \\
\hline TP & 9 & 10 & 13 & \\
\hline
\end{tabular}

Abbreviations: CVC, central venous catheter; PICC, peripherally inserted central catheter; NTC, non-tunneled central venous catheter; GI, gastrointestinal; CAF, cyclophosphamide + anthracycline + fluorouracil; DCF, docetaxel + cisplatin + fluorouracil; FOLFIRI, irinotecan + leucovorin + fluorouracil; FOLFOX, oxaliplatin + leucovorin + fluorouracil; GP, gemcitabine + platinum; GT, gemcitabine + taxane; NP, navelbine + platinum; PF, platinum + fluorouracil; PP, pemetrexed + platinum; TAC, taxane + anthracycline + cyclophosphamide; TF, taxane + fluorouracil; TP, taxane + platinum.

removed due to completion of chemotherapy and four were extubated because of complications. Thirty NTCs were removed due to completion of therapy and 10 were due to complications. A Kaplan-Meier survival analysis curve for time to catheter removal (including completion of therapy, death, and complications) by the type of CVCs was plotted to visualize the survival rate over time (Figure 1).

\section{Catheter-related complications}

There was just one complication in the port group. Twentyfour catheters of PICC groups developed complications, and 11 lines of NTCs developed complications. Port group was associated with the lowest overall complication rate compared with the other two types of CVCs $(P<0.01$ and $P<0.01$, respectively). There was no statistical difference in the number of complications in PICC and NTC groups $(P>0.05)$. The major complications of PICC were mechanical phlebitis and catheter-related thrombosis (75.0\%), and the major complication of NTC was catheter-related infection $(45.5 \%)$. All the catheter-related infections in our study were exit-site local infection. Table 3 shows the detailed complications of the three types of CVCs.

\section{Cost of different types of CVCs}

The average cost of the port was $\$ 1,449.0 \pm 54.3$ in our study. This cost contained the catheter fee, operation-related cost, monthly maintenance cost, and cost of treatment of complications and removal. The average cost of PICC was $\$ 1,089.3 \pm 160.5$, and the NTC cost was $\$ 437.9 \pm 82.0$ on average, including catheter fee, catheterization cost, weekly maintenance cost, and removal and complication treatment cost. There was a significant difference among the three types of CVCs $(P<0.01)$. For different induration times, the average costs were different. When the duration time was $<12$ months, the cost of port was much higher than PICC and NTC ( $P<0.01$ and $P<0.01$, respectively). When the duration time was longer than 12 months, the cost of port had no difference with the cost of PICC (Table 4).

\section{Quality of life and patients' satisfaction}

Patients of port group had higher quality of life and comfort than the other two types of CVCs $(P<0.01$ and $P<0.01$, respectively). Two patients of port group were not investigated on satisfaction because of death. Out of 43 patients in port group, 41 (95.3\%) were satisfied with the catheter. The satisfaction degree of port group was higher than the other two groups ( $P<0.01$ and $P<0.01$, respectively) (Table 5).

\section{Discussion}

This study compared the application of central venous port, peripherally inserted CVC and NTC. To the best of our knowledge, this study is the largest study comparing the three types of CVCs in patients with malignant tumor to guide the

Table 2 The duration time of three types of CVCs

\begin{tabular}{lllllll}
\hline Group & Mean time (days) & $\mathbf{D T} \leq \mathbf{6 M}$ & $\mathbf{6 M}<\mathbf{D T}<\mathbf{I} \mathbf{M}$ & $\mathbf{D T} \geq \mathbf{I} \mathbf{2 M}$ & $\mathbf{n}$ & $\mathbf{P}$-value \\
\hline Port & $338.3 \pm 93.8$ & 2 & 22 & 21 & 45 \\
PICC & $261.1 \pm 63.6$ & 8 & 48 & 4 & 0.000 \\
NTC & $119.9 \pm 33.2$ & 35 & 5 & 0 & 60 & \\
\hline
\end{tabular}

Abbreviations: CVC, central venous catheter; DT, duration time; M, month; PICC, peripherally inserted central catheter; NTC, non-tunneled central venous catheter. 


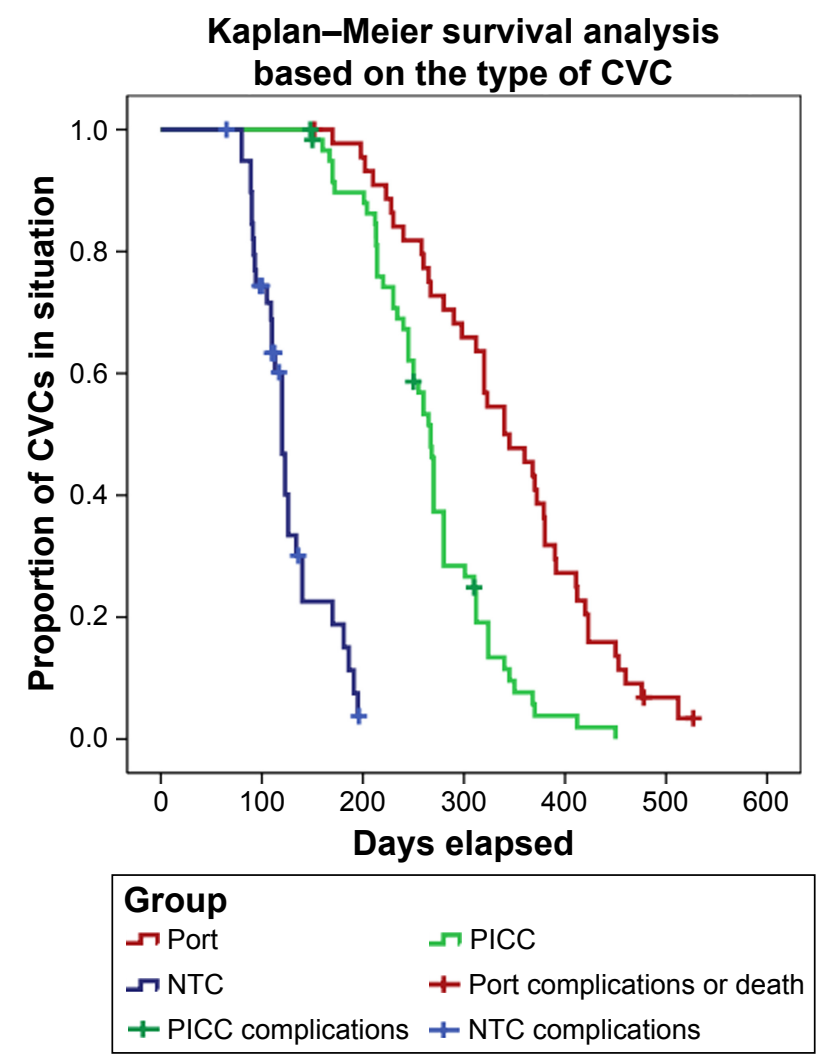

Figure I Kaplan-Meier survival analysis curve for time to catheter removal (all removals including treatment completion, death, and complications) by the type of CVCs.

Abbreviations: CVC, central venous catheter; PICC, peripherally inserted central catheter; NTC, non-tunneled central venous catheter.

clinical preference for CVC use. Our study found that the success rate $(95.6 \%)$ by one puncture in port was the highest in the three types of CVCs. There was no catheterizationrelated complication in our study. Implantation of port was performed in operating rooms with the help of monitoring devices, and this could reduce procedure-related complications, such as pneumothorax, hemothorax, and arterial damage. ${ }^{18}$ Implantation of PICC was performed by professional PICC nurse under B-ultrasound guidance. The success rate by one puncture of port (95.6\%) was higher than the NTC (80\%). Implantation of NTC was conducted by clinical experienced surgeons through the puncture of the internal jugular vein by the Seldinger technique without ultrasound guidance, which may be the reason for the relatively low success rate by one puncture. Ultrasound-guided catheter insertion was reported the lowest proportion of failures in the previous study. ${ }^{19}$ In our study, the mean duration time of ports was 338.36 days (152-527 days), and they were removed due to complications only in one case, similar to Hill's report. ${ }^{20}$ NTCs had the shortest duration time (119.90 days on average), and 10 of 40 (25\%) were removed because of complications. In the former study, ports were also reported having longer duration time and only a few cases removed by complications..$^{21}$ Therefore, ports may be considered an effective tool for the long-term use in patients with cancer. In our study, the NTC was used longer than the recommended time. Usually, NTCs had shorter duration time when they were used in intensive care units or emergency departments. It was reported to have longer duration time when NTCs were used in chemotherapy. ${ }^{8}$ Many patients in our study were positive to express their own preference to retain the catheter to use when there was no complication. There was no CRBSI in our study.

In our study, port group was associated with the lowest overall complication rate compared with the other two types of CVCs $(P<0.01$ and $P<0.01$, respectively), which support previous reports where ports were reported fewer complications compared with PICCs. ${ }^{1,9}$ Only one port developed occlusion complication. Thrombosis was reported as a severe complication of an implanted port, and the reported incidence of thromboembolism related to the catheter was up to $12 \%-64 \% .^{13,22,23}$ We only conducted ultrasound examination for thrombosis when there were thrombosis-related symptoms, which may leave out the asymptomatic catheterrelated thrombosis. There was no statistical difference in the total complication rate between PICC and NTC (40\% vs $27.5 \%, P>0.05)$. Mechanical phlebitis and catheter-related thrombosis $(75 \%)$ were the most common complications of PICCs in our study. Although 10 of 60 PICCs had mechanical phlebitis after PICC insertion, none was removed due to this complication. We gave treatment to the patients who had catheter-related mechanical phlebitis, which may be the reason for no serious consequences. Our treatment included applying mucopolysaccharide polysulfide cream to the skin along the vein accompanied with hot-wet compress. PICCrelated thrombosis was reported high to $51.4 \%,{ }^{5}$ but in our

Table 3 Complications of the three types of CVCs

\begin{tabular}{|c|c|c|c|c|c|c|c|}
\hline Group & Phlebitis & Thrombosis & Infection & Malposition & Occlusion & Breakage & Total, n (\%) \\
\hline Port & 0 & 0 & 0 & 0 & 1 & 0 & I (2.2) \\
\hline PICC & 10 & 8 & 2 & 0 & 3 & I & $24(40)$ \\
\hline NTC & 0 & 2 & 5 & 2 & 2 & 0 & II (27.5) \\
\hline
\end{tabular}

Notes: Port vs PICC, $P<0.0$ I; port vs NTC, $P<0.01$; PICC vs NTC, $P>0.05$.

Abbreviations: CVC, central venous catheter; PICC, peripherally inserted central catheter; NTC, non-tunneled central venous catheter. 
Table 4 Cost of different types of CVCs in different duration times

\begin{tabular}{|c|c|c|c|c|c|c|c|c|}
\hline \multirow[t]{2}{*}{ Group } & \multicolumn{2}{|c|}{ Average cost } & \multicolumn{2}{|c|}{$\begin{array}{l}\text { Average cost for } \\
\text { DT } \leq 6 \mathrm{M}\end{array}$} & \multicolumn{2}{|c|}{$\begin{array}{l}\text { Average cost for } \\
6 \mathrm{M}<\mathrm{DT}<12 \mathrm{M}\end{array}$} & \multicolumn{2}{|c|}{$\begin{array}{l}\text { Average cost for } \\
\text { DT } \geq 12 M\end{array}$} \\
\hline & $\mathbf{n}$ & $\$$ & $\mathbf{n}$ & $\$$ & $n$ & $\$$ & $n$ & $\$$ \\
\hline Port & 45 & I,449.0 \pm 54.3 & 2 & $1,349.2 \pm 2.3$ & 22 & $1,4 \mid 2.4 \pm 27.9$ & 21 & $1,496.8 \pm 28.5$ \\
\hline $\mathrm{PICC}$ & 60 & $1,089.3 \pm 160.5$ & 8 & $838.2 \pm 26.2$ & 48 & $I, 102.1 \pm 102.0$ & 4 & $\mathrm{I}, 437.9 \pm 94.7$ \\
\hline NTC & 40 & $437.9 \pm 82.0$ & 35 & $4 \mid 3.3 \pm 52.1$ & 5 & $609.8 \pm 15.9$ & 0 & NA \\
\hline
\end{tabular}

Notes: Average cost: port vs PICC: $P<0.01$; port vs NTC, $P<0.0$; PICC vs NTC, $P<0.01$. Average cost for DT $\leq 6 \mathrm{M}$ : port vs PICC, $P<0.01$; $P$ ort vs NTC, $P<0.01$; PICC vs NTC, $P<0.01$. Average cost for $6 \mathrm{M}<$ DT $<12 \mathrm{M}$ : port vs PICC, $P<0.01$; port vs NTC, $P<0.01$; PICC vs NTC, $P<0.01$. Average cost for DT $\geq 12 \mathrm{M}$ : port vs PICC, $P>0.05$. Abbreviations: CVC, central venous catheter; DT, duration time; M, month; PICC, peripherally inserted central catheter; NTC, non-tunneled central venous catheter; NA, not available.

study, we only detected the symptomatic thrombosis for the three types of CVCs. The major complication of NTC was catheter-related infection (45.5\%) in our study, and 10 of 40 NTCs were removed because of complications, which contain five cases of catheter-related infection, two cases of catheter malposition, two cases of catheter occlusion, and one case of thrombosis. Compared to port and PICC, NTC had more serious complications. The reason may be that NTC we used was polyurea urethane material, and insertion veins, the jugular veins or subclavian veins, were more prone to sweating. It was reported that risk factors for catheter-related infection could be factors associated with CVC insertion parts, type of CVC, which had great impact on risk of catheter infection. ${ }^{24}$

The average cost of the port was $\$ 1,449.0 \pm 54.3$. PICC cost was $\$ 1,089.3 \pm 160.5$ and NTC cost was $\$ 437.9 \pm 82.0$ in our study. There was a significant difference among the three types of CVCs $(P<0.01)$. Catheter cost of port was self-financed in China, which was much more expensive than PICC and NTC (\$960, \$364, and \$68.4, respectively), while the cost of PICC and NTC could be reimbursed by medical insurance. The maintenance costs for PICC or NTC were about $\$ 75.7$ a month and $\$ 18.9$ for port a month. When the duration time was $<12$ months, the cost of port was much higher than PICC and NTC. When the duration time was longer than 12 months, the cost of port had no difference with the cost of PICC. Many of the patients in this study preferred PICC or NTC instead of port because of the cost. Thus, the economic factor was an important reason that led to fewer catheterizations of port in our study, which was similar to the previous study. ${ }^{8}$ As far as expenses were concerned, our recommendation was that PICC was a good choice for the patients. Compared with port, the same experience was reported. ${ }^{25}$ In Martella et al's ${ }^{25}$ report, the authors supported the utilization of PICCs when the patient was to be treated with trabectedin, and they found that PICCs showed an improved cost-efficiency ratio compared with ports when the device was used for less than a year as it was generally when patients received trabectedin. Compared with NTC, PICC had longer indwelling time and fewer complications that lead to catheter removal. What is more, the maintenance costs for the two types of CVCs were similar. Thus, for patients with treatment less than a year, PICC was a good choice for chemotherapy from an economic point.

Patients of port group had higher quality of life and comfort than the other two types of CVCs, and the satisfaction of port was higher than the other two groups $(P<0.01$ and $P<0.01$, respectively). The patients with port can swim and bath as normal. The maintenance of port is easily compared with PICC and NTC. There is no need of change for dressing around exit sites, only need monthly flushing when not in use, compared with weekly flushing for PICC and NTC. There is no visibility of an external line and a minimal risk of damage or catheter malposition. Acceptability to patients was reported greater for a port. ${ }^{2}$ It was reported that the lack of restriction in patients' daily activities, increased comfort, long-term use capability, and reliability were the greatest advantages of port catheters compared to other central catheters. ${ }^{18,26} \mathrm{It}$ was reported that the upper arm port was with higher quality of life for patients than the chest ports implanted in the

Table 5 Quality of life, comfort, and satisfaction of different types of CVCs

\begin{tabular}{|c|c|c|c|c|c|c|}
\hline Group & Average score & Discomfort & Medium comfort & Comfort & Not satisfied & Satisfied, n (\%) \\
\hline Port & $23.9 \pm 6.1$ & 8 & 10 & 22 & 2 & $4 I(95.3)$ \\
\hline PICC & $|5| \pm 5.7$. & 40 & 14 & 6 & 10 & $50(83.3)$ \\
\hline NTC & $14.2 \pm 4.0$ & 30 & 15 & 0 & 18 & $22(55)$ \\
\hline
\end{tabular}

Notes: Average score, port vs PICC, $P<0.01$; PICC vs NTC, $P<0.01$. Satisfaction, port vs PICC, $P<0.01$; PICC vs NTC, $P<0.01$.

Abbreviations: CVC, central venous catheter; PICC, peripherally inserted central catheter; NTC, non-tunneled central venous catheter. 
jugular vein or the subclavian vein. Patients with upper arm ports do not require upper body undressing, which avoids embarrassment and discomfort, especially in women. ${ }^{13}$ The ports in our study were all chest ports implanted in the jugular vein or the subclavian vein.

There were some limitations in the study. Patients had their own options for the catheter, especially considering the cost factor. What is more, this was an observational study and there was no randomization of patients to the different groups. In the future, a multicenter prospective study with random grouping will be needed to confirm the conclusion.

\section{Conclusion}

Port costs more, has fewer complications, and has a higher quality of life and patients' satisfaction than PICCs and NTCs. Besides complications and procedure compliance, cost, patients' quality of life and comfort can be additional critical issues in the decision-making about which catheter to be used. Despite with less cost, NTC has more complications and shorter duration time and is not suitable for long-term chemotherapy. Medical staff can rely on their experience and patients' characteristics and preference to choose the most appropriate CVC for long-term chemotherapy.

\section{Acknowledgments}

This study was funded by the Weifang Health Development Project of China. We acknowledge the doctors who inserted ports and NTCs and also PICC team nurses in our study.

\section{Disclosure}

The authors report no conflicts of interest in this work.

\section{References}

1. Patel GS, Jain K, Kumar R, et al. Comparison of peripherally inserted central venous catheters (PICC) versus subcutaneously implanted portchamber catheters by complication and cost for patients receiving chemotherapy for non-haematological malignancies. Support Care Cancer. 2014;22(1):121-128.

2. Coady K, Ali M, Sidloff D, Kenningham RR, Ahmed S. A comparison of infections and complications in central venous catheters in adults with solid tumours. J Vasc Access. 2015;16(1):38-41.

3. Loveday HP, Wilson JA, Pratt RJ, et al; UK Department of Health. epic3: national evidence-based guidelines for preventing healthcare-associated infections in NHS hospitals in England. J Hosp Infect. 2014;86(suppl 1): S1-S70.

4. Hill S. Trapezius placement of implanted ports: understanding the procedure. Br J Nurs. 2016;25(suppl 2):S9-S15.

5. Liu Y, Gao Y, Wei L, Chen W, Ma X, Song L. Peripherally inserted central catheter thrombosis incidence and risk factors in cancer patients: a double-center prospective investigation. Ther Clin Risk Manag. 2015; 11:153-160.

6. Groeger JS, Lucas AB, Thaler HT, et al. Infectious morbidity associated with long-term use of venous access devices in patients with cancer. Ann Intern Med. 1993;119(12):1168-1174.
7. Kappers-Klunne MC, Degener JE, Stijnen T, Abels J. Complications from long-term indwelling central venous catheters in hematologic patients with special reference to infection. Cancer. 1989;64(8):1747-1752.

8. Babu KG, Suresh Babu MC, Lokanatha D, Bhat GR. Outcomes, cost comparison, and patient satisfaction during long-term central venous access in cancer patients: experience from a Tertiary Care Cancer Institute in South India. Indian J Med Paediatr Oncol. 2016;37(4):232-238.

9. Worth LJ, Seymour JF, Slavin MA. Infective and thrombotic complications of central venous catheters in patients with hematological malignancy: prospective evaluation of nontunneled devices. Support Care Cancer. 2009;17(7):811-818.

10. O'Grady NP, Alexander M, Burns LA, et al. Guidelines for the prevention of intravascular catheter-related infections. Am J Infect Control. 2011;39(4 suppl 1):S1-S34.

11. Chopra V, O'Horo JC, Rogers MA, Maki DG, Safdar N. The risk of bloodstream infection associated with peripherally inserted central catheters compared with central venous catheters in adults: a systematic review and meta-analysis. Infect Control Hosp Epidemiol. 2013;34(9): 908-918.

12. Zaghal A, Khalife M, Mukherji D, et al. Update on totally implantable venous access devices. Surg Oncol. 2012;21(3):207-215.

13. Li Y, Cai Y, Gan X, et al. Application and comparison of different implanted ports in malignant tumor patients. World J Surg Oncol. 2016; 14(1):251.

14. Mollee P, Jones M, Stackelroth J, et al. Catheter-associated bloodstream infection incidence and risk factors in adults with cancer: a prospective cohort study. J Hosp Infect. 2011;78(1):26-30.

15. Vepsalainen K, Lassila R, Arola M, et al. Complications associated with central venous access device in children with haemophilia: a nationwide multicentre study in Finland. Haemophilia. 2015;21(6):747-753.

16. Li J, Fan Y-Y, Xin M-Z, et al. A randomised, controlled trial comparing the long-term effects of peripherally inserted central catheter placement in chemotherapy patients using B-mode ultrasound with modified Seldinger technique versus blind puncture. Eur J Oncol Nurs. 2014;18(1):94-103.

17. Mori $\mathrm{Y}$, Nagayama S, Kawamura J, et al. A retrospective analysis on the utility and complications of upper arm ports in 433 cases at a single institute. Int J Clin Oncol. 2016;21(3):474-482.

18. Aziret M, Irkorucu O, Gokler C, et al. Performance of venous port catheter insertion by a general surgeon: a prospective study. Int Surg. 2015;100(5):827-835.

19. Biffi R, Orsi F, Pozzi S, et al. Best choice of central venous insertion site for the prevention of catheter-related complications in adult patients who need cancer therapy: a randomized trial. Ann Oncol. 2009; 20(5):935-940.

20. Hill S. Totally implanted ports: the trapezius approach in practice. Br J Nurs. 2015;24(19):S22-S26.

21. Cotogni P, Pittiruti M, Barbero C, Monge T, Palmo A, Boggio Bertinet D. Catheter-related complications in cancer patients on home parenteral nutrition: a prospective study of over 51,000 catheter days. JPEN $J$ Parenter Enteral Nutr. 2013;37(3):375-383.

22. Sutherland DE, Weitz IC, Liebman HA. Thromboembolic complications of cancer: epidemiology, pathogenesis, diagnosis, and treatment. Am J Hematol. 2003;72(1):43-52.

23. Vescia S, Baumgartner AK, Jacobs VR, et al. Management of venous port systems in oncology: a review of current evidence. Ann Oncol. 2008;19(1):9-15.

24. Lu SF, Chen JH, Shang WM, Chou SS. [Prevention and nursing care of central line-associated bloodstream infections in critically ill patients]. Hu Li Za Zhi [J Nurs]. 2012;59(4):5-11. Chinese.

25. Martella F, Salutari V, Marchetti C, et al. A retrospective analysis of trabectedin infusion by peripherally inserted central venous catheters. Anticancer Drugs. 2015;26(9):990-994.

26. Carlo JT, Lamont JP, McCarty TM, Livingston S, Kuhn JA. A prospective randomized trial demonstrating valved implantable ports have fewer complications and lower overall cost than nonvalved implantable ports. Am J Surg. 2004;188(6):722-727. 


\section{Publish your work in this journal}

Patient Preference and Adherence is an international, peer-reviewed, open access journal that focuses on the growing importance of patient preference and adherence throughout the therapeutic continuum. Patient satisfaction, acceptability, quality of life, compliance, persistence and their role in developing new therapeutic modalities and compounds to optimize

clinical outcomes for existing disease states are major areas of interest for the journal. This journal has been accepted for indexing on PubMed Central. The manuscript management system is completely online and includes a very quick and fair peer-review system, which is all easy to use. Visit http://www. dovepress.com/testimonials.php to read real quotes from published authors.

Submit your manuscript here: http://www.dovepress.com/patient-preference-and-adherence-journal 